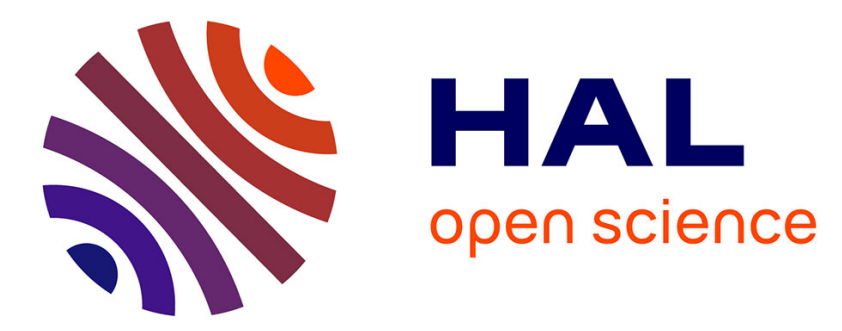

\title{
Mise en évidence et étude des propriétés de transport de nouvelles solutions solides oxyfluorées appartenant au système ternaire $\mathrm{BiF} 3-\mathrm{BiOF}-\mathrm{ThO} 2$
}

\author{
P. Laborde, Jean-Maurice Réau
}

\section{- To cite this version:}

P. Laborde, Jean-Maurice Réau. Mise en évidence et étude des propriétés de transport de nouvelles solutions solides oxyfluorées appartenant au système ternaire $\mathrm{BiF} 3$ - BiOF - ThO2. Journal of Solid State Chemistry, 1988, 72 (2), pp.225-233. 10.1016/0022-4596(88)90026-6 . hal-00260900

\section{HAL Id: hal-00260900 https://hal.science/hal-00260900}

Submitted on 19 Mar 2008

HAL is a multi-disciplinary open access archive for the deposit and dissemination of scientific research documents, whether they are published or not. The documents may come from teaching and research institutions in France or abroad, or from public or private research centers.
L'archive ouverte pluridisciplinaire HAL, est destinée au dépôt et à la diffusion de documents scientifiques de niveau recherche, publiés ou non, émanant des établissements d'enseignement et de recherche français ou étrangers, des laboratoires publics ou privés. 


\title{
Mise en évidence et étude des propriétés de transport de nouvelles solutions solides oxyfluorées appartenant au système ternaire $\mathrm{BiF}_{3}-\mathrm{BiOF}-\mathrm{ThO}_{2}$
}

\author{
P. LABORDE ET J. M. REAU
}

\section{Introduction}

Les fluorures $\operatorname{LnF}_{3}(\operatorname{Ln}=\mathrm{La}, \mathrm{Ce}) \mathrm{de}$ structure type tysonite sont de bons conducteurs anioniques et leurs performances peuvent encore être améliorées par création de lacunes au sein de solutions solides déficitaires en anions formulées $L n_{1-y} B_{y}$ $\mathrm{F}_{3-y}(\mathrm{Ln}=\mathrm{La}, \mathrm{Ce} ; B=\mathrm{Ca}, \mathrm{Sr}, \mathrm{Ba})(1-3)$. Du fait de la polarisabilité élevée de l'ion $\mathrm{Bi}^{3+}\left(3 \AA^{3}\right)(4)$, les matériaux fluorés comportant les meilleurs performances électriques ont été isolés au sein de solutions solides lacunaires à base de $\mathrm{BiF}_{3}: \mathrm{Bi}_{1-y} \mathrm{~K}_{y}$ $\mathrm{F}_{3-2 y}(0,02 \leq y \leq 0,12)(5), \mathrm{Bi}_{1-y} \mathrm{Ba}_{y} \mathrm{~F}_{3-y}$ $(0,05 \leq y \leq 0,17)(6), \mathrm{Bi}_{1-y} \mathrm{~Pb}_{y} \mathrm{~F}_{3-y}(0,075 \leq$ $y \leq 0,175)(6)$.

A la différence des fluorures, les oxyfluorures de structure dérivée du type tysonite n'ont fait l'objet que de rares investi- gations $(7,8)$. Cette classe de matériaux possède pourtant l'avantage d'associer une conductivité de l'ion $\mathrm{F}^{-}$convenable à une stabilité thermique plus élevée. Ainsi le remplacement progressif du fluor par l'oxygène dans $\mathrm{CeF}_{3}$ entraîne-t-il une amélioration des performances électriques avec $y$ croissant au sein de la solutions solide $\mathrm{Ce}_{1-y} \mathrm{Bi}_{y} \mathrm{O}_{y} \mathrm{~F}_{3-2 y}(8)$.

Compte tenu de la polarisabilité élevée de l'ion $\mathrm{Th}^{4+}\left(2,7 \AA^{3}\right)(4)$, nous avons entrepris la recherche d'oxyfluorures lacunaires à base de $\mathrm{BiF}_{3}$ au sein du système $\mathrm{BiF}_{3}$ $\mathrm{ThO}_{2}$. Ces matériaux étaient susceptibles de présenter une structure tysonite et de bonnes performances électriques. L'existence d'une telle phase avait été suggérée lors de l'étude radiocristallographique du système $\left(\mathrm{ThOF}_{2}\right)_{1-y}\left(\mathrm{BiF}_{3}\right)_{y}(9)$, qui avait en effet permis de mettre en évidence la prés- 


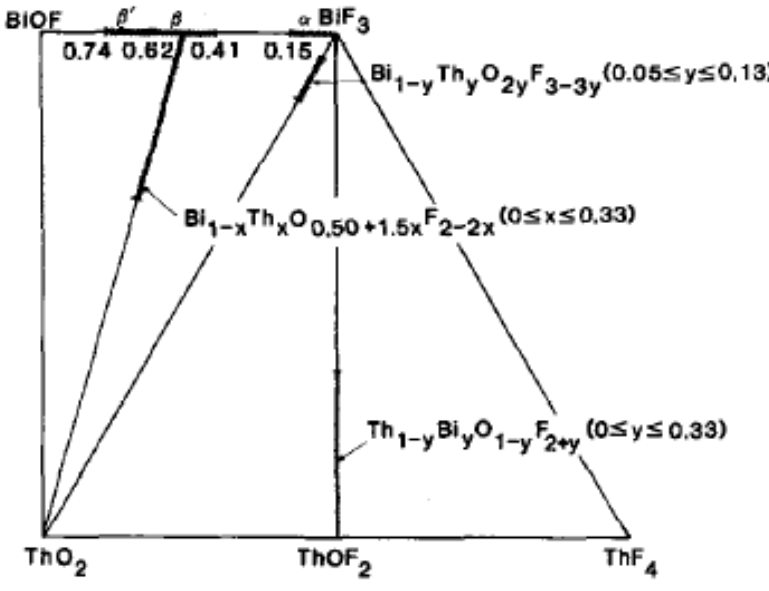

FIG. 1. Phases oxyfluorées étudiées dans le système $\mathrm{ThO}_{2}-\mathrm{ThF}_{4}-\mathrm{BiF}_{3}-\mathrm{BiOF}$.

ence pour $y \geq 0,33$ d'un mélange triphasé constitué de $\mathrm{ThF}_{4}$, de $\mathrm{Th}_{4} \mathrm{Bi}_{2} \mathrm{O}_{4} \mathrm{~F}_{14}$ qui borde la solution solide $\mathrm{Th}_{1-y} \mathrm{Bi}_{y} \mathrm{O}_{1-y} \mathrm{~F}_{2+y}$ $(y=0,33)$ et d'une phase inconnue de type tysonite (fig. 1),

Le système $\mathrm{BiF}_{3}-\mathrm{BiOF}$ comporte un grand nombre de phases de formulation $\mathrm{BiO}_{2} \mathrm{~F}_{3-2 z}$ de structure dérivée du type fluorine ou tysonite (10): $\alpha$ (tysonite) pour $0<z$

\section{TABLEAU I}

INDEXATION DU DIFFRACTOGRAMME $X$ RELATIF À LA COMPOSITION $y=0.10 \mathrm{DE}$ LA SOLUTION SOLIDE $\mathrm{Bi}_{1-y} \mathrm{Th}_{y} \mathrm{O}_{2 y} \mathrm{~F}_{3-3 y}$ $\left(a_{\mathrm{H}}=7,162 \AA, c_{\mathrm{H}}=7,312 \AA\right)$

\begin{tabular}{|c|c|c|c|}
\hline$h k l$ & $d_{\text {calc. }}(\AA)$ & $d_{\text {obs. }}(\AA)$ & $I / I_{0}$ \\
\hline $\begin{array}{lll}0 & 02\end{array}$ & 3,66 & 3,67 & 40 \\
\hline 110 & 3,58 & 3,59 & 35 \\
\hline 111 & 3,216 & 3,225 & 100 \\
\hline 112 & 2,559 & 2,562 & 15 \\
\hline 300 & 2,067 & 2,067 & 75 \\
\hline 113 & 2,016 & 2,016 & 70 \\
\hline 004 & 1,830 & 1,829 & 10 \\
\hline $\begin{array}{lll}302 \\
\end{array}$ & 1,800 & 1,800 & 40 \\
\hline 221 & 1,739 & 1,739 & 20 \\
\hline 114 & 1,629 & 1,629 & 10 \\
\hline 222 & 1,608 & 1,607 & 5 \\
\hline 304 & 1,370 & 1,368 & 10 \\
\hline 115 & 1,355 & 1,353 & 15 \\
\hline
\end{tabular}

$\leq 0,15, \beta$ (fluorine) pour $0,41 \leq z \leq 0,62, \beta^{\prime}$ orthorhombique dérivée de $\beta$ pour $0,62 \leq z$ $\leq 0,74$. Prenant en compte ces résultats, nous avons étudié l'influence de la substitution de l'oxygène au fluor sur les propriétés électriques de $\mathrm{BiO}_{2} \mathrm{~F}_{3-2 z}$ au sein du domaine $\mathrm{BiO}_{z} \mathrm{~F}_{3-2 z}-\mathrm{ThO}_{2}$ (fig. 1). Dans un premier temps nous nous limiterons ici au pseudo-binaire $\mathrm{BiO}_{0,5} \mathrm{~F}_{2}-\mathrm{ThO}_{2}(z=0,50)$.

La solution solide $\mathrm{Bi}_{1-y} \mathrm{Th}_{y} \mathrm{O}_{2 y} \mathrm{~F}_{3-3 y}$ $(0,05 \leq y \leq 0,13)$

\section{Analyse radiocristallographique}

L'analyse radiocristallographique des phases du systeme $\mathrm{BiF}_{3}-\mathrm{ThO}_{2}$ obtenues par trempe à partir de $700^{\circ} \mathrm{C}$ révèle l'existence d'une solution solide de symétrie hexagonale et de formulation $\mathrm{Bi}_{1-y} \mathrm{Th}_{y} \mathrm{O}_{2 y} \mathrm{~F}_{3-3 y}$ $(0,05 \leq y \leq 0,13)$. Sa structure est de type tysonite (fig. 1) (tableau I).

Les paramètres $a_{\mathrm{H}}$ et $c_{\mathrm{H}}$ de la maille élementaire ne varient que très peu avec la composition. La substitution formelle: $\mathrm{Bi}^{3+}$ $+3 \mathrm{~F}^{-}=\mathrm{Th}^{4+}+2 \mathrm{O}^{2-}+\square$ n'entraîne qu'une légère diminution de $c_{H}$ liée au remplacement de $\mathrm{Bi}^{3+}$ par $\mathrm{Th}^{4+}$ de taille légèrement plus faible (fig. 2).

Les limites du domaine de la solution solide $\mathrm{Bi}_{1-y} \mathrm{Th}_{y} \mathrm{O}_{2 y} \mathrm{~F}_{3-3 y}(0,05 \leq y \leq 0,13)$ sont voisines de celles relatives aux solutions

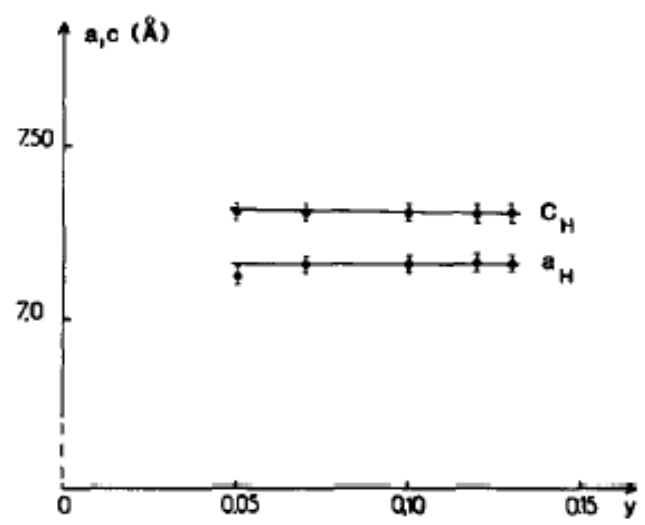

FIG. 2. Variation en fonction de la composition des paramètres $a_{\mathrm{H}}$ et $c_{\mathrm{H}}$ de la maille élémentaire de $\mathrm{Bi}_{1-y}$ $\mathrm{Th}_{y} \mathrm{O}_{2 y} \mathrm{~F}_{3-3 y}(0,05 \leq y \leq 0,13)$. 


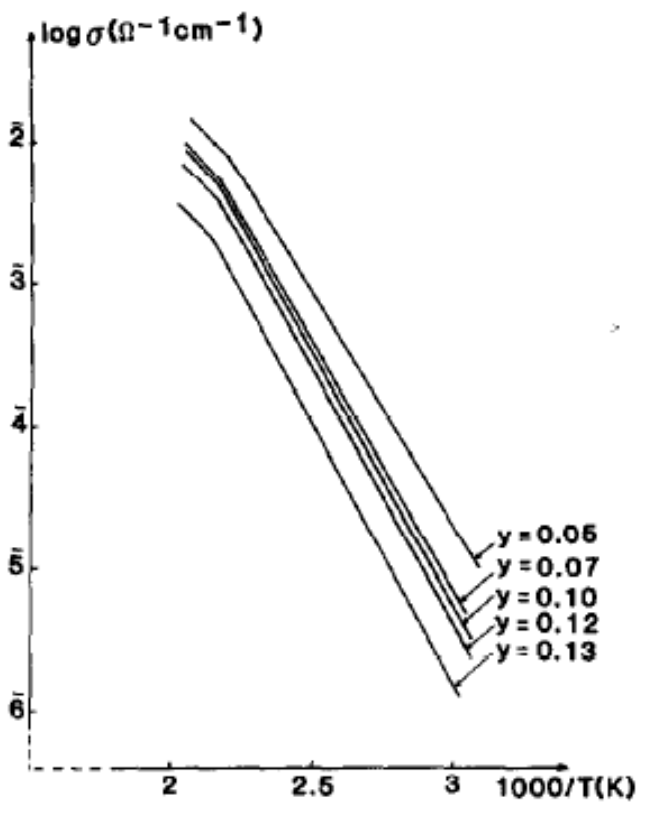

FIG. 3. Variation du logarithme de la conductivité en fonction de l'inverse de la température absolue pour diverses compositions de la solution solide $\mathrm{Bi}_{1-y} \mathrm{Th}_{y}$ $\mathrm{O}_{2 y} \mathrm{~F}_{3-3 y}(0,05 \leq y \leq 0,13)$.

solides $\mathrm{Bi}_{1-y} \mathrm{~K}_{y} \mathrm{~F}_{3-2 y}(0,02 \leq y \leq 0,12)$, $\mathrm{Bi}_{1-y} \mathrm{Ba}_{y} \mathrm{~F}_{3-y}(0,05 \leq y \leq 0,17)$, et $\mathrm{Bi}_{1-y} \mathrm{~Pb}_{y}$ $\mathrm{F}_{3-y}(0,075 \leq y \leq 0,175)$. On peut noter que $\mathrm{BiF}_{3}$ possède la structure tysonite lorsqu'il est stabilisé par un cation de grande taille.

\section{Etude des propriétés étectriques}

La figure 3 donne la variation du logarithme de la conductivité en fonction de l'inverse de la température absolue pour di-

\section{TABLEAU II}

VARIATION DES ÉNERGIES D'ACTIVATION RELATIVES AUX DOMAINES DE BASSE TEMPÉRATURE $\left(\Delta E_{1}\right)$ ET DE hAUTE TEMPÉRATURE $\left(\Delta E_{2}\right)$ ET DE LA TEMPÉRATURE DE CHANGEMENT DE PENTE $t_{\mathrm{p}}$ AVEC $y$

\begin{tabular}{ccccc}
\hline$y$ & $\Delta E_{\mathrm{t}}(\mathrm{eV})$ & $\Delta E_{2}(\mathrm{eV})$ & $\Delta E_{\mathrm{f}}-\Delta E_{2}(\mathrm{eV})$ & $t_{\mathrm{p}}\left({ }^{\circ} \mathrm{C}\right)$ \\
\hline 0,05 & 0,64 & 0,39 & 0,25 & 181 \\
0,07 & 0,68 & 0,42 & 0,26 & 188 \\
0,10 & 0,71 & 0,43 & 0,28 & 190 \\
0,12 & 0,71 & 0,41 & 0,30 & 192 \\
0,13 & 0,72 & 0,42 & 0,30 & 194 \\
\hline
\end{tabular}

verses compositions de la solution solide $\mathrm{Bi}_{1-y} \mathrm{Th}_{y} \mathrm{O}_{2 y} \mathrm{~F}_{3-3 y}(0,05 \leq y \leq 0,13)$.

Dans le domaine de température considéré les compositions $\mathrm{Bi}_{1-y} \mathrm{Th}_{y} \mathrm{O}_{2 y} \mathrm{~F}_{3-3 y}$ présentent deux pentes successives à température croissante, chacune traduisant un comportement de type Arrhénius.

Comme le montre le tableau II, les énergies d'activation relatives au domaine de haute température $\left(\Delta E_{2}\right)$ sont inférieures à celles correspondant au domaine de basse température $\left(\Delta E_{1}\right)$. Ce comportement est analogue à celui observé pour les solutions solides fluorées telles que $L n_{1-y} B_{y} \mathrm{~F}_{3-y}$ ( $L n$ $=\mathrm{La}, \mathrm{Ce} ; B=\mathrm{Ca}, \mathrm{Sr}, \mathrm{Ba})(1,3)$ et $\mathrm{Bi}_{1-y} B_{y}^{\prime}$ $\mathrm{F}_{3-2 y}\left(B^{\prime}=\mathrm{Ba}, \mathrm{Pb}\right)(6)$ ou oxyfluorées telles que $\mathrm{Ce}_{1-y} \mathrm{Bi}_{y} \mathrm{O}_{y} \mathrm{~F}_{3-2 y}(8)$. Par analogie la température du changement de pente qui augmente légèrement avec $y$ (tableau II) peut donc être interprétée comme celle audessus de laquelle existe un échange entre sous-réseaux anioniques.

La figure 4 donne quelques isothermes de

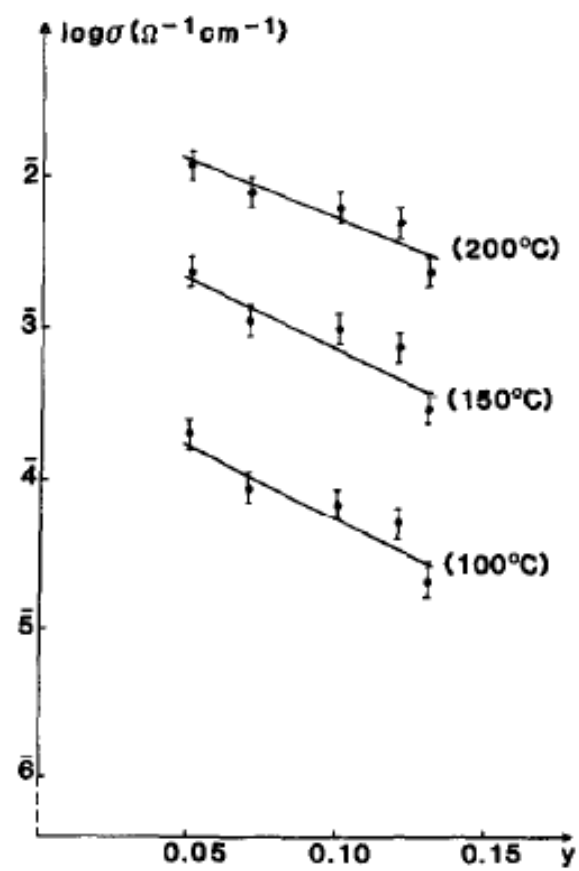

FIc. 4. Isothermes de conductivité relatifs aux domaines de basse température $\left(100\right.$ et $\left.150^{\circ} \mathrm{C}\right)$ et de haute temperature $\left(200^{\circ} \mathrm{C}\right)$ pour $\mathrm{Bi}_{1-y} \mathrm{Th}_{y} \mathrm{O}_{2 y} \mathrm{~F}_{3-3 y}(0,05 \leq y \leq$ $0,13)$. 


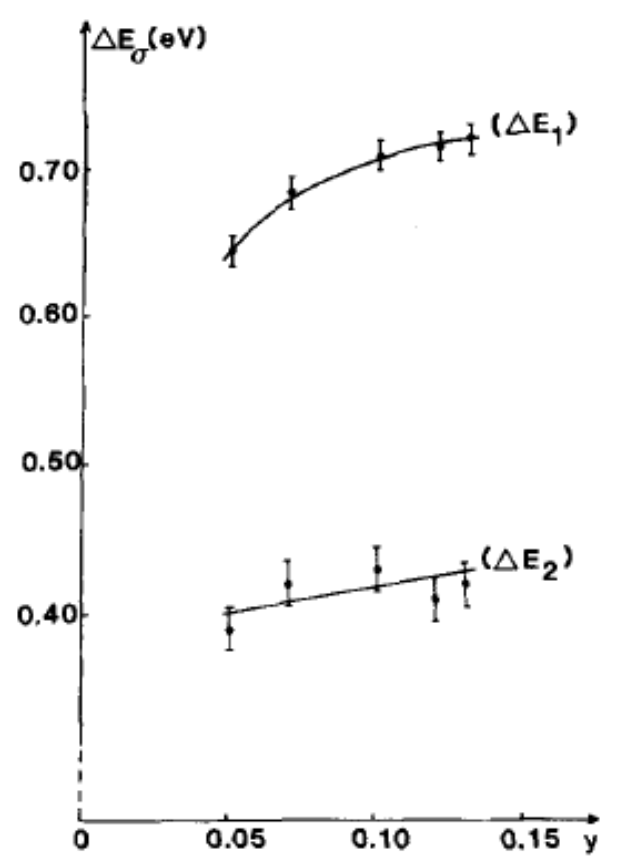

FIG. 5. Variation avec $y$ des énergies d'activation de basse $\left(\Delta E_{1}\right)$ et de haute $\left(\Delta E_{2}\right)$ température pour $\mathrm{Bi}_{1-y}$ $\mathrm{Th}_{y} \mathrm{O}_{2 y} \mathrm{~F}_{3-3 y}$.

conductivité relatifs au domaine de basse température $\left(100\right.$ et $\left.150^{\circ} \mathrm{C}\right)$ ainsi qu'au domaine de haute température $\left(200^{\circ} \mathrm{C}\right)$. La variation avec $y$ des énergies d'activation de basse et de haute température est donnée à la figure 5 .

Quel que soit le domaine de température considéré, $\log \sigma$ diminue régulièrement lorsque $y$ croît. Les énergies d'activation $\Delta E_{1}$ et $\Delta E_{2}$ augmentent progressivement avec $y$. La variation de $\Delta E_{1}$ avec $y$ est supérieure à celle de $\Delta E_{2}$ et il en résulte une augmentation de la différence $\left(\Delta E_{1}-\Delta E_{2}\right)$. Le remplacement du bismuth par le thorium au sein de la solution solide $\mathrm{Bi}_{1-y} \mathrm{Th}_{y}$ $\mathrm{O}_{2 y} \mathrm{~F}_{3-3 y}(0,05 \leq y \leq 0,13)$ entraîne donc un affaiblissement des performances électriques et une température d'échange entre sous-réseaux anioniques plus élevée.

\section{Remarque}

Lors de l'étude des propriétés électriques de la solution solide oxyfluorée $\mathrm{Pb}_{1-x} \mathrm{Bi}_{x} \mathrm{O}_{x}$ $\mathrm{F}_{2-x}$ la mobilité des anions oxygène a été considérée comme négligeable dans le domaine de température étudié $\left(t<250^{\circ} \mathrm{C}\right)$ devant celle des ions $\mathrm{F}^{-}(11)$. Nous avons supposé qu'il en était de même pour $\mathrm{Bi}_{1-y}$ $\mathrm{Th}_{y} \mathrm{O}_{2 y} \mathrm{~F}_{3-3 y}$, les mesures électriques ayant été effectuées dans le même domaine de température $\left(20^{\circ} \mathrm{C}<t<250^{\circ} \mathrm{C}\right)$.

\section{Corrélations entre propriétés électriques et structurales}

Le mécanisme de substitution formel au sein de la solution solide $\mathrm{Bi}_{1-y} \mathrm{Th}_{y} \mathrm{O}_{2 y} \mathrm{~F}_{3-3 y}$ $(0,05 \leq y \leq 0,13)$ est le suivant:

$$
\mathrm{Bi}^{3+}+3 \mathrm{~F}^{-}=\mathrm{Th}^{4+}+2 \mathrm{O}^{2-}+\square .
$$

Lorsque $y$ croît le nombre de lacunes augmente mais le nombre de cations de polarisabilité élevée diminue légèrement. En ne considérant que ces deux critères d'optimisation, ici en opposition, le second semble l'emporter sur le premier puisqu'un affaiblissement des propriétés électriques est observé. Toutefois les lacunes peuvent être bloquées et ne pas participer aux mécanismes de conduction. De plus il semble raisonnable de penser que la substitution de l'oxygène au fluor influence également les propriétés électriques.

Dans le réseau de $\mathrm{LaF}_{3}$ (symétrie trigonale et groupe d'espace $\boldsymbol{P} \overline{3}_{c_{1}}$ ), les anions se répartissent sur trois sous-réseaux anioniques indépendants appelés respectivement $F_{1}, F_{2}, F_{3}$ et contenant les atomes de fluor dans les proportions $12: 4: 2(6,12-$ 15).

L'étude comparée des propriétés structurales de fluorures et d'oxyfluorures de structure tysonite a permis de montrer que l'introduction d'oxygène dans le fluorure entraîne le passage d'une symétrie trigonale $\left(P \overline{3} c_{1}\right)$ à une symétrie hexagónale $\left(P_{6} / \mathrm{mcm}\right)$ et la transformation des trois sous-réseaux anioniques $\mid \mathrm{F}_{1}(12 g), \mathrm{F}_{2}(4 d)$, et $\mathrm{F}_{3}$ (2a) $\mid$ en deux sous-réseaux seulement: $A$ (site $12 k$ ) et $B$ (sites $(4 c)$ et $(2 a)$ ) (8). Les projections des structures de $\mathrm{LaF}_{3}$ et de 


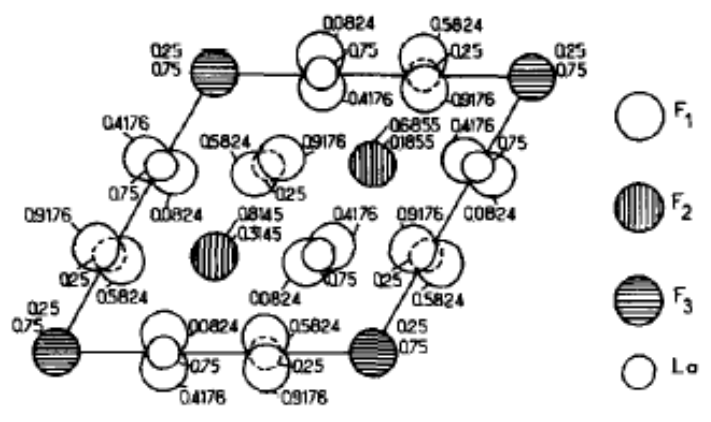

FIG. 6. Projection de la structure de $\mathrm{LaF}_{3}$ sur le plan $x \mathrm{Oy}$.

$\mathrm{ThOF}_{2}$ sur le plan $x \mathrm{O} y$ illustrent ce résultat (figs. 6 et 7 ).

L'étude des propriétés structurales et électriques de la solution solide $\mathrm{Th}_{1-y} \mathrm{Bi}_{y}$ $\mathrm{O}_{1-y} \mathrm{~F}_{2+y}(0 \leq y \leq 0,33)$ a permis d'établir les résultats suivants $(9)$ :

- Le passage de $\mathrm{Th}_{6} \mathrm{O}_{6} \mathrm{~F}_{12}(y=0)$ à $\mathrm{Th}_{4}$ $\mathrm{Bi}_{2} \mathrm{O}_{4} \mathrm{~F}_{14}(y=0,33)$ correspond au remplacement progressif de l'oxygène par le fluor dans les sites $(2 a)$, les sites $(12 k)$ et (4c) restant occupés respectivement par des atomes de fluor et d'oxygène.

- un échange entre les sous-réseaux $A$ et $B$ ne devient possible que lorsque tous les sites (2a) sont occupés par des fluors ( $y=$ $0,33)$.

Il apparaît ainsi que les oxygènes ne peuvent être présents que dans l'un des deux sous-réseaux $A$ et $B$, sinon ils risquent de bloquer les mécanismes de conduction.

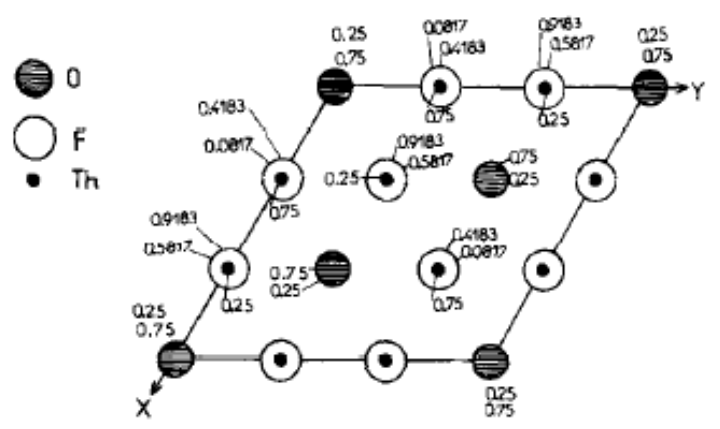

FIG. 7. Projection de la structure de $\mathrm{ThOF}_{2}$ sur le plan $x \mathrm{O} y$.
TABLEAU III

ENERGIES D'ACTIVATION DANS LE DOMAINE DE BASSE TEMPERATURE

$\left(\Delta E_{1}\right)$ RFI.ATIVFS À DIVERSES COMPOSITIONS DES SOLUTIONS SOLIDES FLUORÉES $\mathrm{Bi}_{1-y} B_{y}^{\prime} \mathrm{F}_{3-y}\left(B^{\prime}=\mathrm{Ba}, \mathrm{Pb}\right)$ ET DE LA SOLUTION SOLIDE OXYFLUORÉE $\mathrm{Bi}_{1-y} \mathrm{Th}_{y} \mathrm{O}_{2 y} \mathrm{~F}_{3-3 y}$

\begin{tabular}{lcc}
\hline & $\Delta E_{1}(\mathrm{eV})$ & Ref. \\
\hline $\mathrm{Bi}_{0,90} \mathrm{Ba}_{0,10} \mathrm{~F}_{2,90}$ & 0,58 & $(6)$ \\
$\mathrm{Bi}_{0,85} \mathrm{Ba}_{0,15} \mathrm{~F}_{2,85}$ & 0,56 & $(6)$ \\
$\mathrm{Bi}_{0,955} \mathrm{~Pb}_{0,075} \mathrm{~F}_{2,925}$ & 0,57 & $(6)$ \\
$\mathrm{Bi}_{0,85} \mathrm{~Pb}_{0,15} \mathrm{~F}_{2,85}$ & 0,52 & $(6)$ \\
$\mathrm{Bi}_{0,825} \mathrm{~Pb}_{0,175} \mathrm{~F}_{2,825}$ & 0,51 & $(6)$ \\
$\mathrm{Bi}_{0,95} \mathrm{Th}_{0,05} \mathrm{O}_{0,10} \mathrm{~F}_{2,85}$ & 0,64 & \\
$\mathrm{Bi}_{0,87} \mathrm{Th}_{0,13} \mathrm{O}_{0,26} \mathrm{~F}_{2,61}$ & 0,72 & \\
\hline
\end{tabular}

Cette étude a permis de préciser également qu'à basse température seuls les ions fluor du sous-réseau $A$ de l'oxyfluorure (et par extrapolation les ions fluor du sousréseau $F_{1}$ dans les fluorures) interviennent dans les mécanismes de conduction.

Il $a$ été établi d'autre part à partir de considérations géométriques que dans $\mathrm{LaF}_{3}$ les ions fluor du sous-réseau $F_{2}$ gênaient les mouvements en "dents de scie" des ions fluor $F_{1}$ dans leur propre sous-réseau (6). L'extrapolation de ce résultat aux oxyfluorures nous amène à penser que, compte tenu de leur charge, les ions oxygène placés dans le sous-réseau $B$, même s'ils s'éloignent légèrement des ions fluor du sous-réseau $A$, entraîneront une barrière d'énergie plus élevée pour les ions fluor mobiles du sous-réseau $A$.

Nous avons rassemblé au tableau III les valeurs de l'énergie d'activation dans le domaine de basse température $\left(\Delta E_{1}\right)$ pour diverses compositions des solutions solides fluorées $\mathrm{Bi}_{1-y} B_{y}^{\prime} \mathrm{F}_{3-y}\left(B^{\prime}=\mathrm{Ba}, \mathrm{Pb}\right)$ et de la solution solide oxyfluorée $\mathrm{Bi}_{1-y} \mathrm{Th}_{y} \mathrm{O}_{2 y}$ $\mathrm{F}_{3-3 y} . \Delta E_{1}$ est pratiquement indépendant de $y$ dans $\mathrm{Bi}_{1-y} B_{y}^{\prime} \mathrm{F}_{3-y}$, en revanche $\Delta E_{1}$ augmente régulièrcment avcc $y$ dans $\mathrm{Bi}_{1-y}$ $\mathrm{Th}_{y} \mathrm{O}_{2 y} \mathrm{~F}_{3-3 y}$ (fig. 5). 


\section{TABLEAU IV}

COMPARAISON DES PROPRIETÉS Électriques dE L'OXYFLUORURE $\mathrm{Bi}_{0,95} \mathrm{Th}_{0,05} \mathrm{O}_{0,10} \mathrm{~F}_{2,85}$ à CELLES DE FLUORURES DE MÊME STRUCTURE À BASE DE $\mathrm{LaF}_{3}$, $\mathrm{CeF}_{3}$, ET BiF

\begin{tabular}{lccc}
\hline & $\begin{array}{c}\sigma_{140^{\circ} \mathrm{c}} \\
\left(\Omega^{-1} \mathrm{~cm}^{-1}\right)\end{array}$ & $\begin{array}{c}\Delta E \\
(\mathrm{eV})\end{array}$ & Ref. \\
\hline $\mathrm{La}_{0,93} \mathrm{Ba}_{0,07} \mathrm{~F}_{2,93}$ & $3 \times 10^{-3}$ & 0,38 & $(I)$ \\
$\mathrm{Ce}_{0,95} \mathrm{Ca}_{0,05} \mathrm{~F}_{2,95}$ & $6 \times 10^{-3}$ & 0,37 & $(2)$ \\
$\mathrm{Bi}_{0,85} \mathrm{Ba}_{0,15} \mathrm{~F}_{2,85}$ & $7 \times 10^{-3}$ & 0,56 & $(6)$ \\
$\mathrm{Bi}_{0,85} \mathrm{~Pb}_{0,15} \mathrm{~F}_{2,85}$ & $8 \times 10^{-3}$ & 0,52 & $(6)$ \\
$\mathrm{Bi}_{0,94} \mathrm{~K}_{0,06} \mathrm{~F}_{2,88}$ & $2 \times 10^{-2}$ & 0,28 & $(5)$ \\
$\mathrm{Bi}_{0,95} \mathrm{Th}_{0,05} \mathrm{O}_{0,10} \mathrm{~F}_{2,85}$ & $2 \times 10^{-3}$ & 0,64 & \\
\hline
\end{tabular}

Toutes ces considérations nous conduisent, en accord avec la variation des propriétés électriques, à placer dans $\mathrm{Bi}_{1-y}$ $\mathrm{Th}_{y} \mathrm{O}_{2 y} \mathrm{~F}_{3-3 y}$ les ions oxygène au sein du sous-réseau $B$.

Le mécanisme de substitution proposé donne naissance également à des lacunes, soit dans l'un des deux sous-réseaux $A$ ou $B$ soit dans les deux. Il a été proposé dans le cas des solutions solides fluorées de type tysonite déficitaires en anions, sur la base de considérations structurales et énergétiques, que le sous-réseau lacunaire serait le sous-réseau $\mathrm{F}_{2}(6,16)$. L'extrapolation de ce résultat à la solution solide oxyfluorée $\mathrm{Bi}_{1-y} \mathrm{Th}_{y} \mathrm{O}_{2 y} \mathrm{~F}_{3-3 y}$ conduirait alors à la formation de lacunes avec $y$ croissant dans le sous-réseau $B$. Dans une telle éventualité les lacunes libérées resteraient bloquées au sein de ce sous-réseau et ne participeraient pas aux mécanismes de conduction à basse température.

Dans ces conditions la formule développée de la composition limite $(y=0,13)$ de la solution solide $\mathrm{Bi}_{1-y} \mathrm{Th}_{y} \mathrm{O}_{2 y} \mathrm{~F}_{3-3 y}$ pourrait s'écrire:

$$
\mathrm{Bi}_{5,22} \mathrm{Th}_{0,78}\left|\mathrm{~F}_{12}\right|_{A}\left|\mathrm{~F}_{3,66} \mathrm{O}_{1,56} \square_{0,78}\right|_{B} .
$$

Vu la faible proportion d'oxygène, même pour $y=0,13$, cette hypothèse de répartition serait en accord avec la variation des propriétés électriques avec la température: quel que soit $y$, un échange entre sous-réseaux anioniques est mis en évidence pour $175^{\circ} \mathrm{C}<t<190^{\circ} \mathrm{C}$.

Une étude par simulation de la stabilité de la composition " $\mathrm{Bi}_{5} \mathrm{ThO}_{2} \mathrm{~F}_{15}$ "' (soit $y=$ $0,167)$ qui est proche de la limite $y=0,13$ de la solution solide $\mathrm{Bi}_{1-y} \mathrm{Th}_{y} \mathrm{O}_{2 y} \mathrm{~F}_{3-3 y}$ pourrait permettre de confirmer la présence des atomes d'oxygène et des lacunes dans le sousréseau $B$.

Les propriétés électriques de l'oxyfluorure $\mathrm{Bi}_{0,95} \mathrm{Th}_{0,05} \mathrm{O}_{0,10} \mathrm{~F}_{2,8}$ s sont comparées au tableau IV à celles de fluorures de même structure à base de $\mathrm{LaF}_{3}, \mathrm{CeF}_{3}$, et $\mathrm{BiF}_{3}: \mathrm{Bi}_{0,95} \mathrm{Th}_{0,05} \mathrm{O}_{0,10} \mathrm{~F}_{2,85}$ se place parmi les meilleurs conducteurs connus de l'ion $\mathrm{F}^{-}$à structure tysonite ou dérivée.

$$
\begin{aligned}
& \text { La solution solide } \mathrm{Bi}_{1-x} \mathrm{Th}_{x} \mathrm{O}_{0,5+1,5 x} \mathrm{~F}_{2-2 x} \\
& (0 \leq x \leq 0,33)
\end{aligned}
$$

\section{Analyse radiocristallographique}

L'analyse radiocristallographique des phases du pseudobinaire $\mathrm{BiO}_{0,5} \mathrm{~F}_{2}-\mathrm{ThO}_{2}$ obtenues par trempe à partir de $900^{\circ} \mathrm{C}$ révèle l'existence d'un domaine de solution solide de formulation $\mathrm{Bi}_{1-x} \mathrm{Th}_{x} \mathrm{O}_{0,5+1,5 x} \mathrm{~F}_{2-2 x}$ $(0 \leq x \leq 0,33)$, dont la structure dérive du type fluorine (Fig. 1).

Le mécanisme de substitution formel au sein de cette solution solide est le suivant:

$$
2 \mathrm{Bi}^{3+}+4 \mathrm{~F}^{-}=2 \mathrm{Th}^{4+}+3 \mathrm{O}^{2-}+\square .
$$

Le remplacement de deux atomes de bismuth par deux atomes de thorium serait lié à la substitution de trois atomcs d'oxygène à quatre atomes de fluor de manière à assurer la neutralité électrique, elle entraînerait donc la liberation d'une lacune anionique. Ce mécanisme de substitution ne donne naissance qu'à une faible contraction de la maille élémentaire, liée à la substitution de $\mathrm{Bi}^{3+}$ par $\mathrm{Th}^{4+}$ de taille légèrement plus faible (fig. 8).

\section{Etude des propriétés électriques}

La figure 9 représente la variation du lo- 


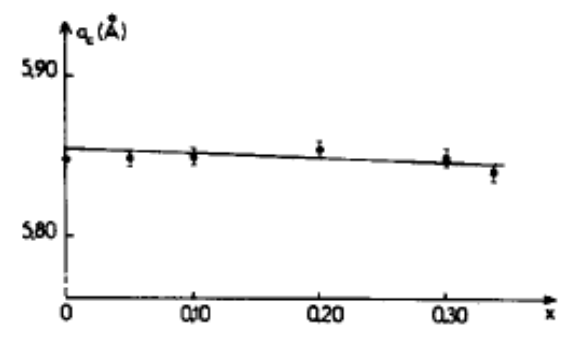

FIG. 8. Variation du paramètre de maille élémentaire $a_{\mathrm{c}}$ en fonction de la composition $x$ au sein de la solution solide $\mathrm{Bi}_{1-x} \mathrm{Th}_{x} \mathrm{O}_{0,5+1,5 x} \mathrm{~F}_{2-2 x}(0 \leq x \leq 0,33)$.

garithme de la conductivité en fonction de l'inverse de la température pour quelques compositions de la solution solide $\mathrm{Bi}_{1-x} \mathrm{Th}_{x}$ $\mathrm{O}_{0,5+1,5 x} \mathrm{~F}_{2-2 x}(0 \leq x \leq 0,33)$. Dans le domaine de température considéré, la conductivité suit une loi d'Arrhénius: $\sigma=$ $\sigma_{0} \exp \left(-\Delta E_{\sigma} / k T\right)$, où $\Delta E_{\sigma}$ représente l'énergie d'activation relative aux mouvements diffusifs des porteurs.

Les variations de la conductivité à $t=60$ et $150^{\circ} \mathrm{C}$ et de l'énergie d'activation $\Delta E_{\sigma}$

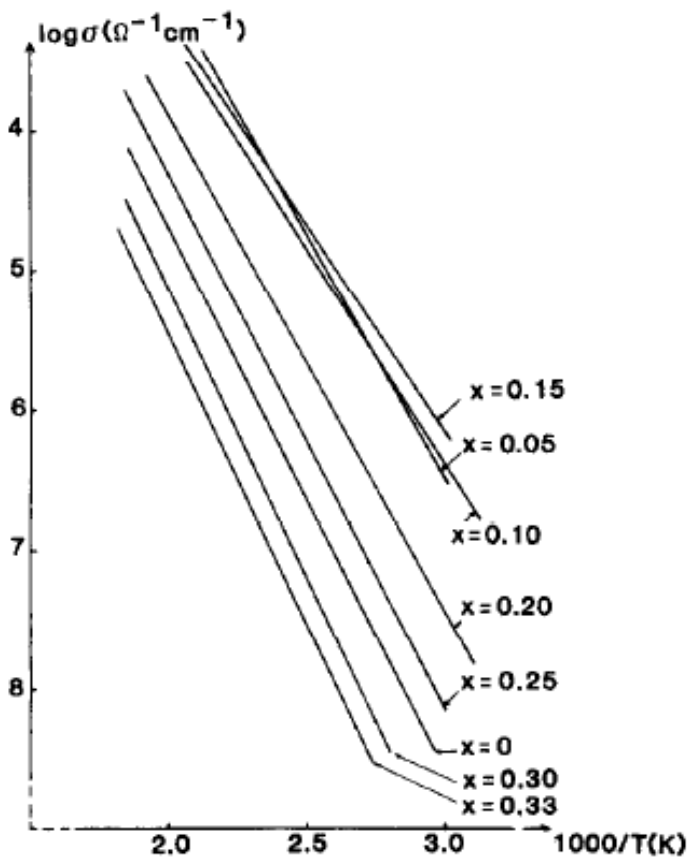

FIG. 9. Variation du logarithme de la conductivité en fonction de l'inverse de la température pour diverses compositions de la solution solide $\mathrm{Bi}_{1-x} \mathrm{Th}_{x} \mathrm{O}_{0,5+1,5 x}$ $\mathrm{F}_{2-2 x}(0 \leq x \leq 0,33)$.

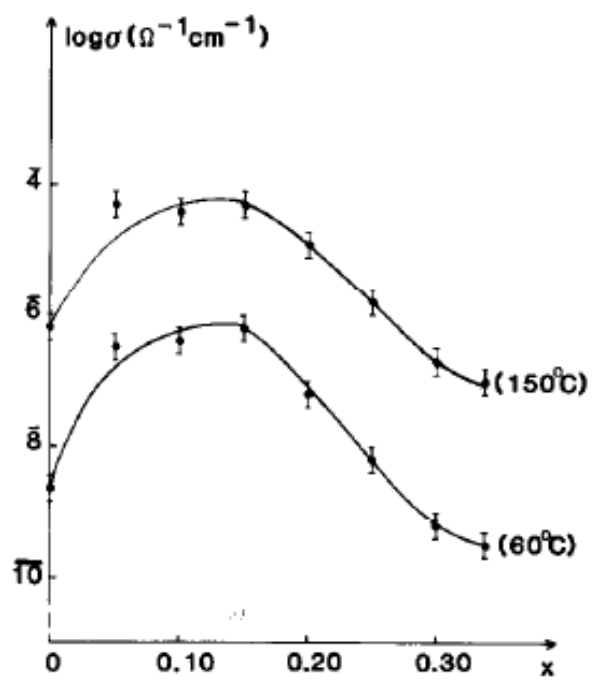

FIG. 10. Variation avec $x$ de la conductivité à $t=60$ et $150^{\circ} \mathrm{C}$ au sein de la solution solide $\mathrm{Bi}_{1-x} \mathrm{Th}_{x} \mathrm{O}_{0,5+1,5 x}$ $\mathrm{F}_{2-2 x}(0 \leq x \leq 0,33)$.

avec la composition sont données dans les figures 10 et 11 .

Lorsque $x$ croît au sein de la solution solide $\mathrm{Bi}_{1-x} \mathrm{Th}_{x} \mathrm{O}_{0,5+1,5 x} \mathrm{~F}_{2-2 x}$ dans le domaine de composition $0<x \leqslant 0,15$, on observe une diminution rapide de l'énergie d'activation et une augmentation sensible de la conductivité. En revanche pour $x \geq 0,15$, l'énergie d'activation augmente et la conductivité diminue. Il existe donc un maximum de conductivité lié à un minimum

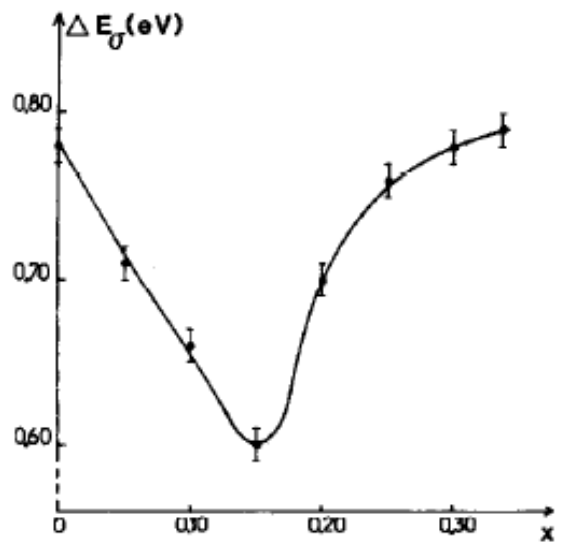

FIG, 11. Variation de l'énergie d'activation avec $x$ pour la solution solide $\mathrm{Bi}_{1-x} \mathrm{Th}_{x} \mathrm{O}_{0,5+1,5 x} \mathrm{~F}_{2-2 x}(0 \leq x \leq$ 0,33). 
d'énergie d'activation pour $x \simeq 0,15$, soit pour $x \simeq x_{\mathrm{L} / 2}, x_{\mathrm{L}}$ représentant la limite extrême de la solution solide.

\section{Corrélations entre propriétés électriques et structurales}

Lorsque $x$ augmente au sein de la solution solide $\mathrm{Bi}_{1-x} \mathrm{Th}_{x} \mathrm{O}_{0,5+1,5 x} \mathrm{~F}_{2-2 x}(0 \leq x \leq$ 0,33 ), le remplacement d'un atome de bismuth par un atome de thorium de polarisabilité légèrement plus faible $\left(\alpha_{\mathrm{Bi}^{3}},=3 \AA^{3}\right.$, $\alpha_{\mathrm{Th}^{4+}}=2,7 \AA^{3}(4)$ ) devrait entraîner un affaiblissement des propriétés électriques, ce qui n'est observé que pour $x \geqslant 0,15$. Lc critère "polarisabilité cationique" semble donc n'avoir qu'une influence mineure sur la variation des propriétés électriques lorsque les cations mis en jeu sont de polarisabilité voisine.

La variation des propriétés électriques avec la composition de $\mathrm{Bi}_{1-x} \mathrm{Th}_{x} \mathrm{O}_{0,5+1,5 x}$ $\mathrm{F}_{2-2 x}$ est analogue à celle des solutions solides $\mathrm{Pb}_{1-x} \mathrm{Bi}_{x} \mathrm{~F}_{2+x}(0 \leq x \leq 0,50)$ et $\mathrm{Pb}_{1-x}$ $\operatorname{Th}_{x} \mathrm{~F}_{2+2 x}(0 \leq x \leq 0,25)$ qui comportent chacune un maximum de conductivité associé à un minimum d'énergie d'activation pour $x$ $\approx x_{\mathrm{L} / 2}, x_{\mathrm{L}}$ étant la limite extrême de la solution solide; l'étude de ces solutions solides par diffraction de neutrons a montré qu'un maximum de désordre apparaît pour les compositions relatives à $x \simeq x_{\mathrm{L} / 2}(17,18)$.

Les deux compositions limites de la solution solide $\mathrm{Bi}_{1-x} \mathrm{Th}_{x} \mathrm{O}_{0,5+1,5 x} \mathrm{~F}_{2-2 x}(0 \leq x \leq$ 0,33 ) répondent respectivement aux formules $\mathrm{BiO}_{0,50} \mathrm{~F}_{2}$ et $\mathrm{Bi}_{0,66} \mathrm{Th}_{0,33} \mathrm{OF}_{1,33}$, le passage de la première composition à la seconde correspondant à une diminution du nombre total d'anions $X(X=\mathrm{O}, \mathrm{F})$ : on passe de $X_{2,50}$ à $X_{2,33}$. Elles sont caractérisées par des valeurs particulières du rapport oxygène/fluor égales respectivement à $1 / 4$ et $3 / 4$. De plus $\mathrm{Bi}_{0,66} \mathrm{Th}_{0,33} \mathrm{OF}_{1,33} \mathrm{com}$ porte un rapport bismuth/thorium égal à 2 . Bien que ces matériaux soient désordonnés à grande distance, on peut supposer l'existence d'un ordre à courte distance relativement élevé. Une étude par diffraction de neutrons permettra de le confirmer. Un tel résultat a déjà été mis en évidence pour la limite $\mathrm{Pb}_{0,50} \mathrm{Bi}_{0,50} \mathrm{~F}_{2,50}$ de $\mathrm{Pb}_{\mathrm{t}-x} \mathrm{Bi}_{x} \mathrm{~F}_{2+x}$ (I7).

Le modèle de substitution formel pour $\mathrm{Bi}_{1-x} \mathrm{Th}_{x} \mathrm{O}_{0,5+1,5 x} \mathrm{~F}_{2-2 x}\left(2 \mathrm{Bi}^{3+}+4 \mathrm{~F}^{-}=\right.$ $2 \mathrm{Th}^{4+}+3 \mathrm{O}^{2-}+\square$ ) impose l'augmentation du nombre de lacunes lorsque $x$ croît. On peut supposer que $\mathrm{BiO}_{0,50} \mathrm{~F}_{2}$ comporte un nombre important d'ions fluor en position interstitielle et que le remplacement dans ce matériau de $\mathrm{Bi}^{3+}$ par $\mathrm{Th}^{4+}$ qui engendre un désordre cationique, entraîne simultanément la diminution du nombre d'ions fluor en positions interstitielles et l'augmentation du nombre de lacunes en sites normaux. Il en résulterait un accroissement du désordre et de ce fait une amélioration avec $\mathrm{x}$ croissant des propriétés de transport.

Partant de l'autre limite $\mathrm{Bi}_{0,66} \mathbf{T h}_{0,33} \mathrm{OF}_{1,33}$, le remplacement dans ce matériau de deux $\mathrm{Th}^{4+}$ par deux $\mathrm{Bi}^{3+}$ entraîne a priori l'introduction d'un ion fluor dans un site oxygéné vide. Cependant, on peut supposer que, comme dans $\mathrm{Pb}_{1-x} \mathrm{Bi}_{x} \mathrm{O}_{x} \mathrm{~F}_{2-x}(11)$, les ions fluor ont tendance à se placer préférentiellement en position interstitielle. Il en résulterait alors une augmentation simultanée du nombre de lacunes, du nombre d'ions fluor en position interstitielle, un désordre accru et l'amélioration des propriétés électriques.

Les meilleures performances sont ainsi obtenues pour la composition particulière $\mathrm{Bi}_{0,85} \mathrm{Th}_{0,15} \mathrm{O}_{0,725} \mathrm{~F}_{1,70}$, correspondant à $x=$ 0,15 soit $x_{\mathrm{L} / 2}$, qui devrait comporter un maximum de désordre. Une étude par diffraction de neutrons de $\mathrm{Bi}_{1-x} \mathrm{Th}_{x} \mathrm{O}_{0,5+1,5 x}$ $\mathrm{F}_{2-2 x}$ devrait permettre de confirmer l'ensemble de ces hypothèses.

\section{Conclusions}

L'étude de l'influence respective des divers critères d'optimisation des propriétés de transport au sein de la solution solide oxyfluorée $Y_{1-x} \mathrm{Bi}_{x} \mathrm{OF}(0,52 \leq x \leq 0,95)$ de structure dérivée du type fluorine avait 
montré que la polarisabilité cationique est le critère le plus important lorsque les cations ont des polarisabilités bien distinctes (19). En revanche une investigation des propriétés électriques de la solution solide $\mathrm{Bi}_{1}{ }_{x} \mathrm{Th}_{x} \mathrm{O}_{0,5+1,5 x} \mathrm{~F}_{2-2 x}(0 \leq x \leq 0,33)$ met nettement en évidence l'influence de l'ordre local lorsque les cations sont de polarisabilité voisine.

L'influence de ces critères a également été discutée pour une phase oxyfluorée originale de structure tysonite et de formulation $\mathrm{Bi}_{1-y} \mathrm{Th}_{y} \mathrm{O}_{2 y} \mathrm{~F}_{3-3 y}(0,05 \leq y \leq 0,13)$. Une hypothèse de répartition anionique a été proposée en accord avec la variation thermique des propriétés de transport: les oxygènes et les lacunes se placeraient dans le sous-réseau anionique $B$ du réseau de type $\mathrm{ThOF}_{2}$. Les performances de $\mathrm{Bi}_{0,95}$ $\mathrm{Th}_{0,05} \mathrm{O}_{0,10} \mathrm{~F}_{2,85}$ placent ce matériau parmi les meilleurs conducteurs de l'ion $\mathrm{F}^{-}$de type tysonite.

\section{References}

1. A. Roos, A. F. Aalders, J. Schoonman, A. F. M. ARTS, ET N. W. DE WIN, Solid State Ionics 9-10, 571 (1983).

2. T. TAKahashi, H. IWahara, et T. Ishikawa, $J$. Electrochem. Soc. 124, 280 (1977).

3. H. Geiger, G. Schon, et H. Stork, Solid State Ionics 15, 155 (1985).

4. R. D. Shannon, Acta Crystallogr. Sect. A 32, 751 (1976).
5. M. W. Shafer, G. V. Chandrashekhar, et R. A. Figat, Solid State Ionics 5, 633 (1981).

6. A. Rhandour, J. M. Reau, S. Matar, S. B. Tian, et P. HagenMUller, Mater. Res. Bull. 20, 1309 (1985).

7. J. Schoonman, G. J. Dirksen, et R. W. Bonne, Solid State Comm. 19, 783 (1976).

8. A. Rhandour, J. M. Reau, S. Matar, et P. Hagenmuller, J. Solid State Chem. 64, 206 (1986).

9. A. Rhandour, J. M. Reau, et P. HagenmulLER, J. Solid State Chem., en cours de parution.

10. A. Morell, B. Tanguy, et J. Portier, Bull. Soc. Chim. Fr. 7, 2502 (1971).

11. J. L. Soubeyroux, S. F. Matar, J. M. Reau, et P. Hagenmuller, Solid State Ionics 14, 337 (1984).

12. A. K. Cheetham, B. E. F. Fender, H. Fuess, ET A. F. Wright, Acta Crystallogr. Sect. B 32, 94 (1976).

13. I. Brach ET H. Schulz, Solid State Ionics 15, 135 (1985).

14. B. Maximov et H. Schulz, Acta Crystallogr. Sect. B 41, 88 (1985).

15. A. Zalkin et D. H. Templeton, Acta Crystallogr. Sect. B 41, 91 (1985).

I6. J. R. IgEl, M. C. WINTERSgILl, J. J. FonTANella, A. V. Chadwick, C. G. Andeen, et V. E. Bean, J. Phys. C 15, 7215 (1982).

17. C. Lucat, J. Portier, J. M. Reau, P. HagenMUller, ET J. L. SoUbeyrouX, J. Solid State Chem. 32, 279 (1980).

18. J. L. Soubeyroux, J. M. Reau, S. Matar, C. LuCAT, ET P. HaGenMuller, Solid State Ionics 2, 215 (1981).

19. P. Laborde, J. M. Reau, S. F. Matar, et P. Hagenmuller, Mater. Res. Bull. 20, 1501 (1985). 